02

\title{
Процессы захвата и диссоциативного захвата одного электрона при взаимодействии двухзарядных ионов с молекулами СО
}

\author{
(C) А.А. Басалаев, ${ }^{1}$ В.В. Кузьмичев, ${ }^{1,2}$ М.Н. Панов, ${ }^{1}$ О.В. Смирнов ${ }^{1,3}$ \\ ${ }^{1}$ Физико-технический институт им. А.Ф. Иоффре РАН, \\ 194021 Санкт-Петербург, Россия \\ ${ }^{2}$ Санкт-Петербургский политехнический университет Петра Великого, \\ 194064 Санкт-Петербург, Россия \\ ${ }^{3}$ Санкт-Петербургский Академический университет - научно-образовательный центр нанотехнологий РАН, \\ 195220 Санкт-Петербург, Россия \\ e-mail: a.basalaev@mail.ioffe.ru
}

Поступило в Редакцию 8 февраля 2018 г.

В окончательной редакции 28 ноября 2018 г.

Принято к публикации 4 марта 2019 г.

Измерены абсолютные величины сечений процессов одноэлектронного захвата и одноэлектронного захвата с диссоциацией при взаимодействии ионов $\mathrm{He}^{2+}, \mathrm{C}^{2+}, \mathrm{N}^{2+}$ и $\mathrm{O}^{2+}$ с молекулой СО в диапазоне энергий налетающих ионов от 6.4 до $36.4 \mathrm{keV}$. Обнаружено, что для иона-снаряда $\mathrm{O}^{2+}$ сечение процесса диссоциативного захвата существенно больше, чем сечение процесса одноэлектронного захвата. Дано качественное объяснение этого эффекта.

Ключевые слова: двухзарядные ионы, захват одного электрона, диссоциация однозарядных молекулярных ионов атмосферных газов.

DOI: $10.21883 / J T F .2019 .08 .47886 .53-18$

\section{Введение}

При медленных ион-молекулярных столкновениях, когда скорость налетающего иона $V_{p}$ меньше скорости валентных электронов молекулы-мишени, основным по величине сечения процессом изменения зарядового состояния сталкивающихся частиц является процесс захвата одного электрона (процессы (1) и (2)), например, [1,2] и ссылки в них. Сечение процесса диссоциативного захвата одного электрона у молекул $\mathrm{CO}$, в результате которого образуется либо ион $\mathrm{C}^{+}$и атом $\mathrm{O}$ (процесс $(2 \mathrm{a})$ ), либо ион $\mathrm{O}^{+}$и атом $\mathrm{C}$ (процесс (2б)) при взаимодействии с ионами $\mathrm{H}^{+}$(в области скоростей сближения частиц $V_{p}<1$ a.u.) [3,4], $\operatorname{Ar}^{+}$( $V_{p}=0.08-0.19$ a.u. $), \mathrm{Kr}^{+}\left(V_{p}=0.05-0.11\right.$ a.u. $)$ и $\mathrm{Xe}^{+}\left(V_{p}=0.04-0.07\right.$ a.u.) [5], а так же с ионами $\mathrm{He}^{2+}$ $\left(V_{p}<1\right.$ a.u.) [4] составляет примерно $15 \%$ от сечения процесса одноэлектронного захвата (процесс 1), в котором образуется ион $\mathrm{CO}^{+}$. Этот результат хорошо согласуется с аналогичными данными по ионизации молекулы CO электронами с энергией от 40 до $600 \mathrm{eV}[6,7]$. Однако при столкновении ионов $\mathrm{Ne}^{+}\left(V_{p}=0.1-0.3\right.$ a.u.) с молекулами СО сечение процесса (1) примерно в 2 раза меньше сечения процесса диссоциативного захвата $[8]$.

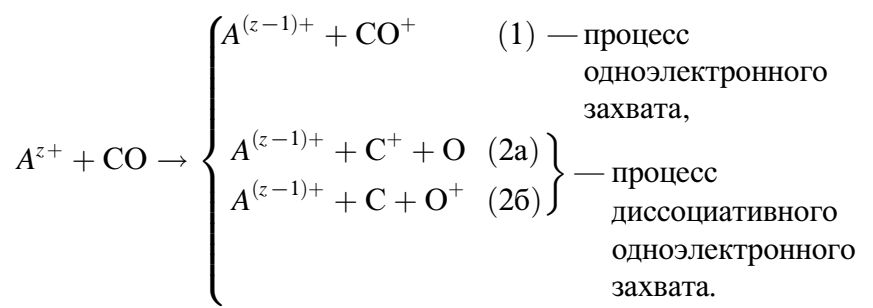

Характерная особенность процесса захвата одного электрона заключается в том, что он эффективно происходит при больших параметрах удара, т.е. при малых углах рассеяния налетающего иона. Величина дифференциального сечения захвата одного электрона при столкновении ионов $\mathrm{H}^{+}$и $\mathrm{He}^{2+}$ с атомами аргона $[9,10]$ и ионов $\mathrm{H}^{+}$с молекулами $\mathrm{CO}$ и $\mathrm{CO}_{2}[11]$ при углах рассеяния налетающей частицы $E \theta=3 \mathrm{keV} \cdot \operatorname{deg}$ (где $\theta-$ угол рассеяния налетающего иона в лабораторной системе координат, $E$ - энергия налетающего иона) падает примерно на 3 порядка. Процесс диссоциативного одноэлектронного захвата при таких углах рассеяния налетающей частицы обусловлен изменением внутренней энергии сталкивающихся частиц, а не кинетической энергией, переданной налетающим ионом атомам, входящим в состав молекулы $\mathrm{CO}$, которая среди двухатомных молекул имеет самую большую энергию диссоциации в основном состоянии $\mathrm{CO}\left(X^{1} \Sigma^{+}\right) D_{0}=11.11 \mathrm{eV}$, а в ионизированном $\mathrm{CO}^{+}\left(X^{2} \Sigma^{+}\right) D_{0}=8.37 \mathrm{eV}$, уступая только молекулярному иону $\mathrm{N}_{2}^{+}\left(X^{2} \Sigma^{+}\right) D_{0}=8.73 \mathrm{eV}[12]$.

Целью настоящей работы было измерение абсолютных величин сечений одноэлектронного захвата и диссоциативного электронного захвата для ион-молекулярных систем $A^{2+}+\mathrm{CO}$, в которых предполагалась значительная роль процесса диссоциативного захвата.

\section{Методика эксперимента}

Для проведения измерений была использована экспериментальная методика, разработанная для исследования фрагментации ионов многоатомных моле- 


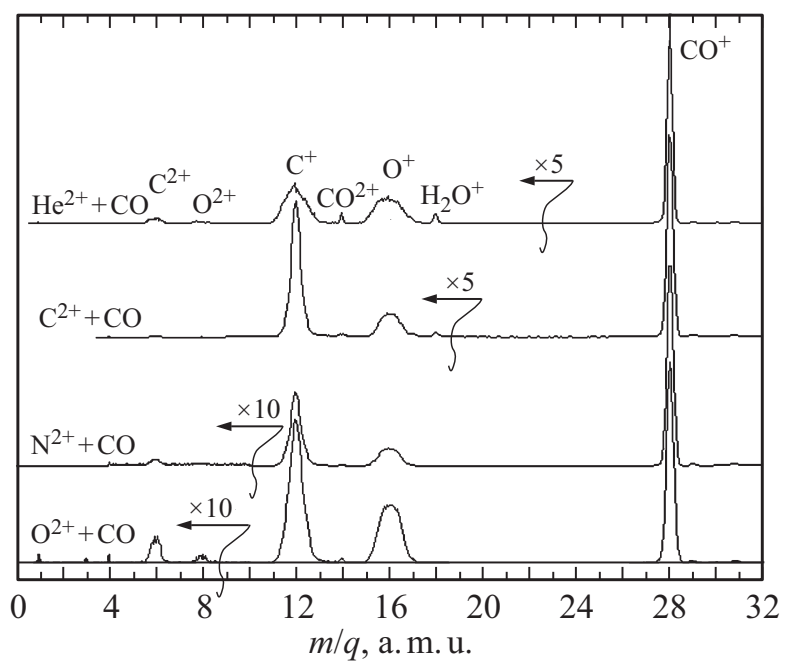

Рис. 1. Типичные масс-спектры, сформированные процессами захвата одного электрона, в которых образовался только один ион отдачи. Все спектры нормированы на один и тот же интеграл пика $\mathrm{CO}^{+}$. Для систем $\mathrm{He}^{2+}+\mathrm{CO}$ и $\mathrm{C}^{2+}+\mathrm{CO}$ области масс-спектров с $m / q<19$ умножены на 5 , для систем $\mathrm{N}^{2+}+\mathrm{CO}$ и $\mathrm{O}^{2+}+\mathrm{CO}$ области масс-спектров с $m / q<10$ умножены на 10 .

кул [13] Хорошо коллимированный $\left(\mathrm{FWHM}=8^{\prime}\right)$ монокинетический пучок двухзарядных ионов с энергией $E_{p}=6.4-36.4 \mathrm{keV}$ пересекал эффузионную струю молекул СО. Пересечение ионного пучка и молекулярной струи происходило в области однородного электрического поля вытягивающего конденсатора, которое направляло ионы отдачи, образовавшиеся в газе-мишени, в ионнооптическую систему времяпролетного масс-анализатора. Эти ионы, пройдя оптическую систему масс-анализатора, имели энергию $4 q \mathrm{keV}$, где $q$ - заряд иона отдачи, и дополнительно ускорялись напряжением $14 \mathrm{kV}$, после чего регистрировались детектором в счетном режиме. Благодаря дополнительному ускорению достигалась высокая и практически одинаковая эффективность регистрации ионов различной массы и заряда.

Зарядовое состояние налетающих ионов, рассеянных в диапазоне углов $\theta= \pm 1.5^{\circ}$, после взаимодействия определялось электростатическим анализатором. Импульсы детектора налетающих ионов $A^{+}$, образовавшихся после захвата одного электрона двухзарядными ионами $A^{2+}$ $(A-$ атомы $\mathrm{He}, \mathrm{C}, \mathrm{N}$ и $\mathrm{O})$, использовались в качестве стартового сигнала регистрирующей системы. Сигналы детектора времяпролетного масс-спектрометра регистрировались в „многостоповом“ режиме, т.е. в режиме, позволяющем зафиксировать разницу времени между стартовым сигналом и временами детектирования всех зарегистрированных ионов-фрагментов. Масс-спектрометр обеспечивал полный сбор ионов-фрагментов с начальными энергиями от 0 до $9 \mathrm{eV}$.

Определение относительных величин сечений процессов одноэлектронного захвата и диссоциативного одноэлектронного захвата проводилось на основе анализа масс-спектров, обусловленных процессами, в которых образовались либо молекулярный ион, либо один заряженный атомарный фрагмент (рис. 1). Таким образом, были разделены процессы одноэлектронного захвата (образовался только один заряженный фрагмент) и захвата электрона с ионизацией (образовались два заряженных фрагмента или двухзарядные ионы $\mathrm{CO}^{2+}, \mathrm{C}^{2+}$, $\left.\mathrm{O}^{2+}\right)$. Для получения величин относительных сечений проводилось интегрирование соответствующих пиков в масс-спектрах и их нормирование на интеграл тока пучка налетающих ионов. Измерения для различных налетающих ионов проводились при неизменном потоке газа-мишени, поэтому полученные величины относительных сечений для всех налетающих ионов были в одинаковых относительных единицах.

Определение абсолютных величин сечений требуют измерения плотности и протяженности струйной молекулярной мишени, что было выполнено с помощью процедуры нормировки измеренного относительного сечения образования ионов $\mathrm{CO}^{+}$при взаимодействии ионов $\mathrm{H}^{+}$(протонов) с молекулами $\mathrm{CO}$ на абсолютное сечение этого процесса, приведенное в работе [3]. Экспериментальные ошибки определения относительных сечений определялись по результатам многократных измерений и не превышали $\pm 2 \%$. Таким образом, ошибка в определении сечений определялась точностью абсолютной калибровки $\pm 10 \%$.

\section{Результаты и их обсуждение}

Сечения процессов одноэлектронного захвата $\sigma_{01}^{21}\left(\mathrm{CO}^{+}\right)$, диссоциативного одноэлектронного захвата $\sigma_{01}^{21}\left(\mathrm{C}^{+}+\mathrm{O}\right)$ и $\sigma_{01}^{21}\left(\mathrm{C}+\mathrm{O}^{+}\right)$и полное сечение захвата одного электрона $\sigma^{21}$, представляющее собой сумму сечений всех элементарных процессов, в которых

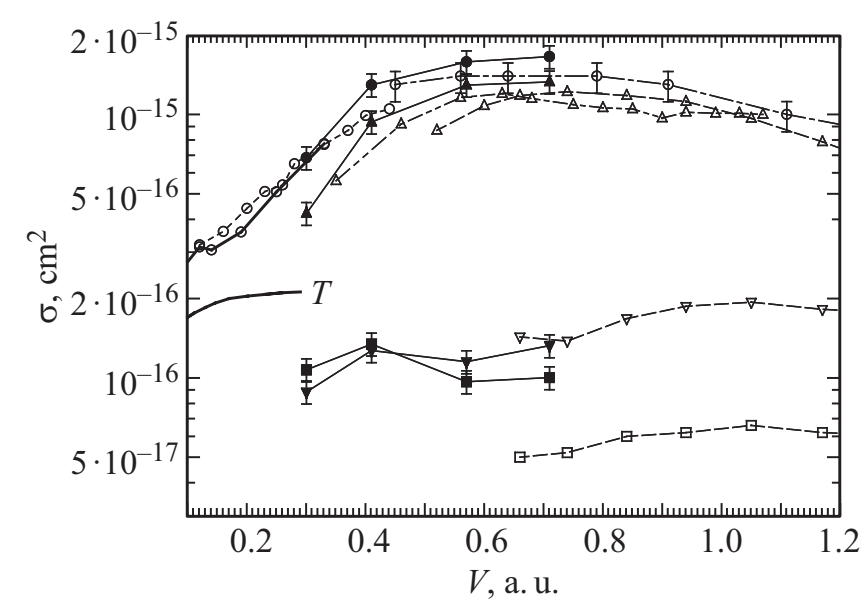

Рис. 2. Сечения процессов одноэлектронного захвата и диссоциативного одноэлектронного захвата при столкновении $\mathrm{He}^{2+}+\mathrm{CO} . \bullet-\sigma^{21}, \boldsymbol{\Delta}-\sigma_{01}^{21}\left(\mathrm{CO}^{+}\right), \quad \boldsymbol{\nabla}-\sigma_{01}^{21}\left(\mathrm{C}^{+}+\mathrm{O}\right)$, $\mathbf{\square}-\sigma_{01}^{21}\left(\mathrm{C}+\mathrm{O}^{+}\right)$настоящая работа; - - $\triangle-\sigma_{01}^{21}\left(\mathrm{CO}^{+}\right)$, $\nabla \quad \sigma_{01}^{21}\left(\mathrm{C}^{+}+\mathrm{O}\right), \quad \square-\sigma_{01}^{21}\left(\mathrm{C}+\mathrm{O}^{+}\right)[4] ; \quad \cdots \Delta^{-\cdots}-$ $\sigma_{01}^{21}\left(\mathrm{CO}^{+}\right)[16] ;-\cdots-\cdots-\sigma^{21}[2], \cdots \odot \cdots-\sigma^{21}[14] ;-\infty$ и $-T-\sigma^{21}$ (эксперимент и расчет соответственно) [15]. 
налетающий ион захватывает один электрон у молекулы мишени, приведены на рис. 2. Измеренное в настоящей работе сечение $\sigma^{21}$ хорошо согласуется с данными других авторов $[2,14,15]$, что позволяет сделать вывод о корректности примененной в работе процедуры определения абсолютных величин сечений.

В работе [16] было проведено масс-спектрометрическое исследование состава ионов отдачи, образующихся при взаимодействии ионов $\mathrm{He}^{2+}$ с молекулами $\mathrm{CO}$. В связи с тем, что авторы работы не фиксировали зарядовое состояние налетающего иона после столкновения, приведенные в работе сечения определяются суммой процессов ионизации, захвата одного и двух электронов, причем сечение последнего $\sigma^{20}$ сравнимо с сечением $\sigma^{21}[2,14,15]$. Однако образование молекулярного иона $\mathrm{CO}^{+}$возможно в процессах одноэлектронного захвата (1) и однократной ионизации, сечение которой в исследуемом диапазоне скоростей должно быть невелико, что и подтверждается хорошим согласием сечения образования иона $\mathrm{CO}^{+}$, полученного в работе [4], с сечением $\sigma_{01}^{21}\left(\mathrm{CO}^{+}\right)$, измеренным в настоящей работе (рис. 2). Рассчитанное методом сильной связи в квазимолекулярном приближении в работе [15] сечение процесса захвата одного электрона существенно меньше экспериментально измеренных как $\sigma^{21}$, так и $\sigma_{01}^{21}\left(\mathrm{CO}^{+}\right)$.

При взаимодействии ионов $\mathrm{C}^{2+}$ с молекулами $\mathrm{CO}$ роль процесса диссоциативного захвата существенно возрастает (рис. 3). При этом сечение процесса (2a) оказывается существенно больше, чем сечение процесса (2б), что характерно для этого процесса при захвате одного электрона протонами [3,4] и для процесса ионизации молекулы СО электронным ударом [6,7]. Расчеты сечения процесса (1), выполненные в работах $[17,18]$, заметно расходятся между собой, но при этом могут считаться разумной оценкой величины сечения одноэлектронного захвата (рис. 3).

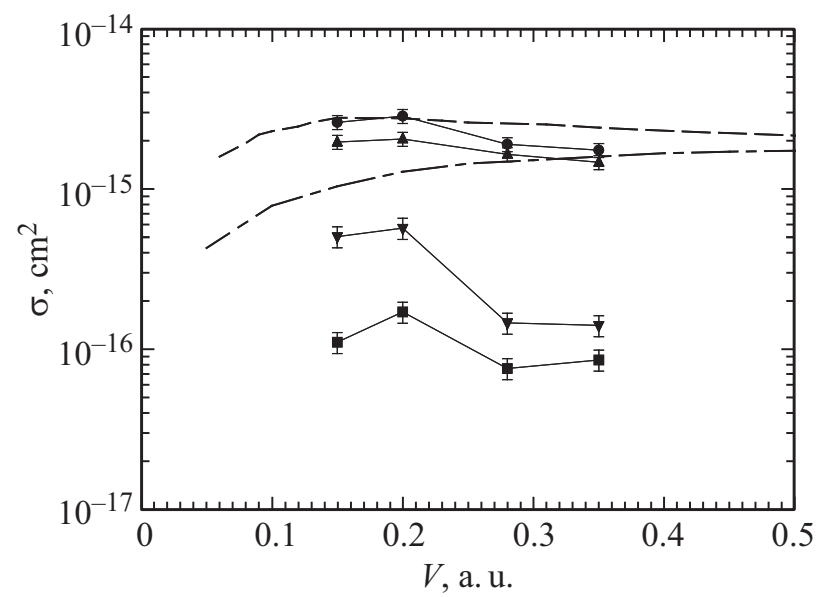

Рис. 3. Сечения процессов одноэлектронного захвата и диссоциативного одноэлектронного захвата при столкновении $\mathrm{C}^{2+}+\mathrm{CO} . \bullet-\sigma^{21}, \boldsymbol{\Delta}-\sigma_{01}^{21}\left(\mathrm{CO}^{+}\right), \boldsymbol{\nabla}-\sigma_{01}^{21}\left(\mathrm{C}^{+}+\mathrm{O}\right)$, - $-\sigma_{01}^{21}\left(\mathrm{C}+\mathrm{O}^{+}\right)$настоящая работа; - - - - [17]; $---[18]$.

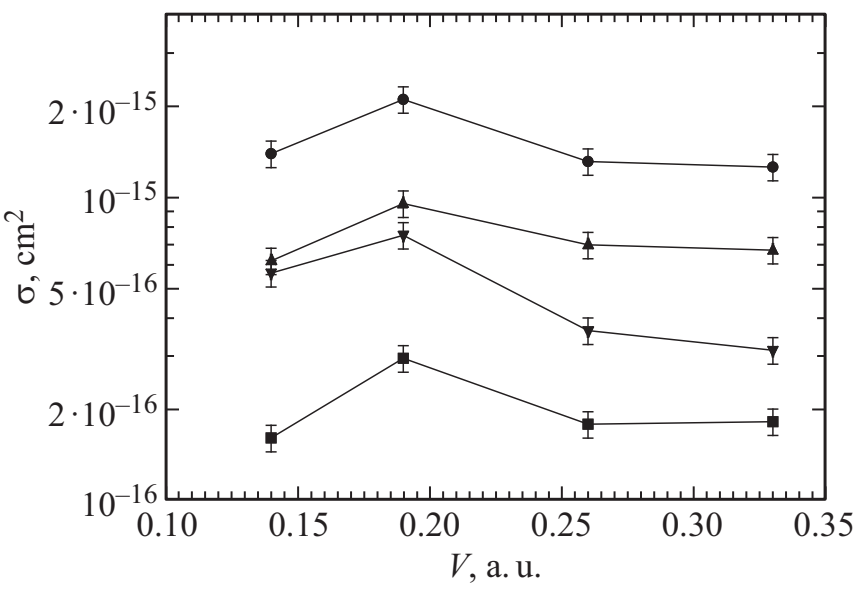

Рис. 4. Сечения процессов одноэлектронного захвата и диссоциативного одноэлектронного захвата при столкновении $\mathrm{N}^{2+}+\mathrm{CO} . \bullet-\sigma^{21}, \boldsymbol{\Delta}-\sigma_{01}^{21}\left(\mathrm{CO}^{+}\right), \boldsymbol{\nabla}-\sigma_{01}^{21}\left(\mathrm{C}^{+}+\mathrm{O}\right), \mathbf{\square}-$ $\sigma_{01}^{21}\left(\mathrm{C}+\mathrm{O}^{+}\right)$настоящая работа.

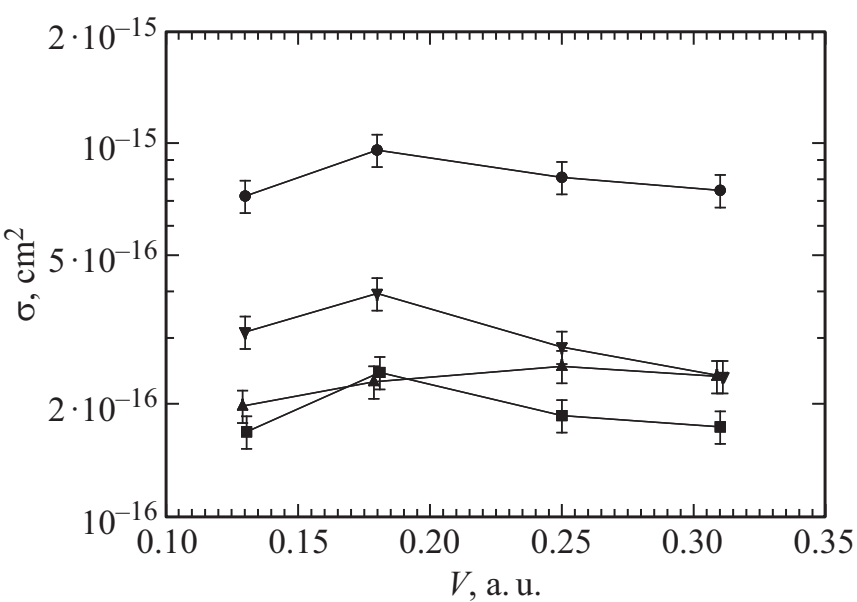

Рис. 5. Сечения процессов одноэлектронного захвата и диссоциативного одноэлектронного захвата при столкновении $\mathrm{O}^{2+}+\mathrm{CO} . \bullet-\sigma^{21}, \boldsymbol{\Delta}-\sigma_{01}^{21}\left(\mathrm{CO}^{+}\right), \boldsymbol{\nabla}-\sigma_{01}^{21}\left(\mathrm{C}^{+}+\mathrm{O}\right), \mathbf{\square}-$ $\sigma_{01}^{21}\left(\mathrm{C}+\mathrm{O}^{+}\right)$настоящая работа.

При взаимодействии ионов $\mathrm{N}^{2+}$ с молекулами $\mathrm{CO}$ процесс (1) также является основным по величине сечения, но при малых скоростях столкновений процесс (2a) имеет сечение, сравнимое по величине с сечением процесса (1) (рис. 4). При взаимодействии ионов $\mathrm{O}^{2+}$ с молекулами $\mathrm{CO}$ сечение процесса диссоциативного захвата (сумма сечений (2a) и (2б)) больше сечения процесса (1) во всем исследованном диапазоне скоростей столкновений (рис. 5), а основным по величине сечения является процесс $(2 \mathrm{a})$.

Дефект энергии экзотермического процесса захвата одного электрона является основным параметром многоуровневой модели Ландау-Зинера [19], в рамках которой обычно рассматривается процесс захвата электрона при взаимодействии многозарядных ионов с атомами, и 
Процессы, происходящие при захвате одного электрона у молекулы СО и соответствующие энергии появления (AE)

\begin{tabular}{c|c|c}
\hline $\begin{array}{c}\text { Обозначение } \\
\text { процесса на } \\
\text { рис. 6 }\end{array}$ & $\begin{array}{c}\text { Продукты } \\
\text { реакции }\end{array}$ & $\mathrm{AE}(\mathrm{eV})$ \\
\hline & $\mathrm{C}\left({ }^{3} \mathrm{P}\right)+\mathrm{O}\left({ }^{3} P\right)$ & $11.11^{a}$ \\
$X$ & $\mathrm{CO}^{+}\left(X^{2} \Sigma^{+}\right)$ & $14.0^{b}$ \\
$A$ & $\mathrm{CO}^{+}\left(A^{2} \Pi\right)$ & $16.5^{b}$ \\
$B$ & $\mathrm{CO}^{+}\left(B^{2} \Sigma^{+}\right)$ & $19.7^{b}$ \\
$C$ & $\mathrm{CO}^{+}\left(C^{2} \Delta\right)$ & $21.8^{b}$ \\
$D$ & $\mathrm{CO}^{+}\left(D{ }^{2} \Pi\right)$ & $22.0^{b}$ \\
1 & $\mathrm{C}^{+}\left({ }^{2} P\right)+\mathrm{O}\left({ }^{3} P\right)$ & $22.4^{c}$ \\
2 & $\mathrm{C}^{+}\left({ }^{2} P\right)+\mathrm{O}\left({ }^{1} D\right)$ & $24.3^{c}$ \\
3 & $\mathrm{O}^{+}\left({ }^{4} S\right)+\mathrm{C}\left({ }^{3} P\right)$ & $24.7^{c}$ \\
4 & $\mathrm{C}^{+}\left({ }^{2} P\right)+\mathrm{O}\left({ }^{1} S\right)$ & $26.6^{c}$ \\
5 & $\mathrm{C}^{+}\left({ }^{4} P\right)+\mathrm{O}\left({ }^{3} P\right)$ & $27.7^{c}$ \\
6 & $\mathrm{O}^{+}\left({ }^{2} D\right)+C\left({ }^{3} P\right)$ & $28.1^{c}$
\end{tabular}

Пр и ме ч ан и е. ${ }^{a}-$ энергия диссоциации молекулы $\mathrm{CO}\left(\mathrm{X}^{1} \Sigma^{+}\right)[12]$, $b$ - энергия молекулярных состояний с $v=0$ [22], ${ }^{c}$ - оценка асимптотической энергии на основании данных об энергии диссоциации [12] и данных об энергии атомных состояний [24].

определяется выражением (3):

$$
Q=E(\operatorname{CO}(X))-E\left(A^{+}\left({ }^{2 s+1} L\right)\right)-E_{\mathrm{ex}}\left(\mathrm{CO}^{+}\left({ }^{2 s+1} \Lambda\right)\right),
$$

где $E(\mathrm{CO}(X))$ - электронная энергия молекулы до столкновения, $E\left(A^{+}\left({ }^{2 s+1} L\right)\right)$ - электронная энергия иона, захватившего электрон, $E_{\mathrm{ex}}\left(\mathrm{CO}^{+}\left({ }^{2 s+1} \Lambda\right)\right)-$ энергия возбуждения образовавшегося молекулярного иона. Процесс экзотермический, если величина $Q>0$.

Для вычисления $Q$ надо знать энергии возможных конечных электронных состояний молекулярного иона $\mathrm{CO}^{+}\left({ }^{2 s+1} \Lambda\right)$, которые были определены в работах [20-22] и измерены экспериментально, например [23]. Асимптотические значения энергий различных каналов диссоциации иона $\mathrm{CO}^{+}$могут быть вычислены на основании данных об энергии атомарных уровней [24] и энергии диссоциации молекулы СО [12], результаты такой оценки хорошо согласуются с экспериментальными [23] и теоретическими данными [21,22]. Молекулярные продукты, образующиеся в процессе захвата одного электрона, и их энергии появления, используемые в настоящей работе для оценки величины $Q$, приведены в таблице.

Процесс захвата электрона многозарядными ионами у атомов эффективно происходит вследствие ландау-зинеровских переходов в области псевдопересечений термов образующейся квазимолекулы. При рассматриваемых скоростях столкновений процесс имеет большое сечение, если $R_{c} \approx 3-9$ a.u. [25], где $R_{c}-$ оценка расстояния сближения сталкивающихся частиц, при котором и происходит электронный переход. Это так называемое „окно реакции“ для налетающих двухзарядных ионов соответствует дефекту энергии экзотермического процесса $Q \approx 3-9 \mathrm{eV}$.
При взаимодействии иона $\mathrm{He}^{2+}$ с молекулой $\mathrm{CO}$ канал процесса захвата электрона в основное состояние иона $\mathrm{He}^{+}(n=1)$ имеет большой дефект энергии $Q \sim 40-30 \mathrm{eV}$, что делает этот канал процесса маловероятным и, скорее всего, при его осуществлении происходит диссоциация образующегося молекулярного иона $\mathrm{CO}^{+}$. Каналы процесса захвата электрона в возбужденное состояние иона $\mathrm{He}^{+}(n=2)$ оказываются эндотермическими, причем минимальный дефект энергии $Q=-0.54 \mathrm{eV}$ имеет канал процесса захвата с образованием недиссоциированного молекулярного иона
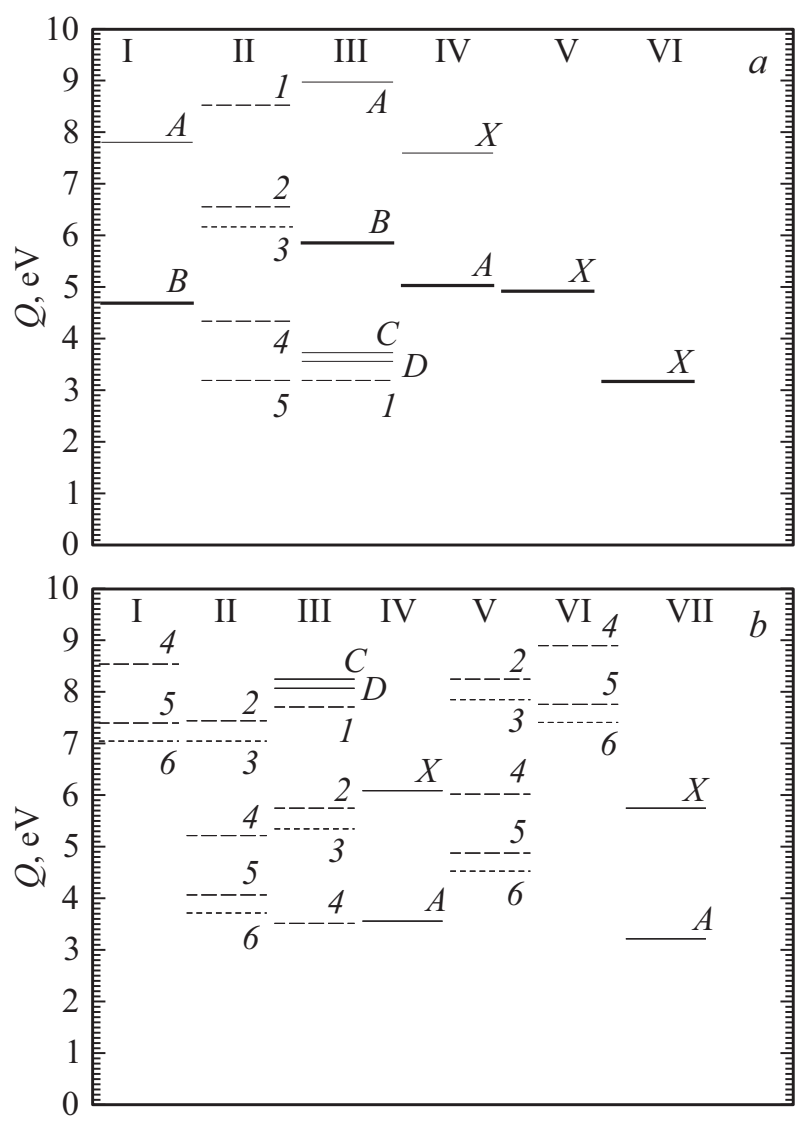

Рис. 6. Дефект энергии процесса захвата одного электрона ионами $\mathrm{C}^{2+}$ и $\mathrm{O}^{2+}$. Индексы у уровней дефектов энергий соответствуют обозначению процессов, приведенных в таблице. $a-\mathrm{C}^{2+}+\mathrm{CO}\left(\mathrm{X}^{1} \Sigma^{+}\right)$. Римскими цифрами обозначены каналы процесса захвата, соответствующие следующим начальным и конечным электронным состояниям налетающего иона: $\mathrm{I}-\mathrm{C}^{2+}\left(2 s^{2}{ }^{1} S\right) \rightarrow \mathrm{C}^{+}\left(2 s^{2} 2 p{ }^{2} P\right)$, II $\mathrm{C}^{2+}\left(2 s 2 p{ }^{3} P\right) \rightarrow \mathrm{C}^{+}\left(2 s^{2} 2 p{ }^{2} P\right), \quad$ III $-\mathrm{C}^{2+}\left(2 s 2 p{ }^{3} P\right) \rightarrow$ $\mathrm{C}^{+}\left(2 s 2 p^{2}{ }^{4} P\right), \mathrm{IV}-\mathrm{C}^{2+}\left(2 s 2 p{ }^{3} P\right) \rightarrow \mathrm{C}^{+}\left(2 s 2 p^{2}{ }^{2} D\right), \mathrm{V}-$ $\mathrm{C}^{2+}\left(2 s^{2} p{ }^{3} P\right) \rightarrow \mathrm{C}^{+}\left(2 s 2 p^{2}{ }^{2} S\right), \quad \mathrm{VI}-\mathrm{C}^{2+}\left(2 s 2 p^{2}{ }^{2} P\right) \rightarrow$ $\mathrm{C}^{+}\left(2 s 2 p^{2}{ }^{2} P\right)$. - - процессы с образованием ионов $\mathrm{CO}^{+}$ (жирными линями отмечены дефекты энергий процессов, наблюдавшихся в работе [26]), ------ - процессы с образованием $\mathrm{C}^{+}+\mathrm{O}, \quad$ процессы с образованием $\mathrm{C}+\mathrm{O}^{+}$. $b-\mathrm{O}^{2+}+\mathrm{CO}\left(X^{1} \Sigma^{+}\right) . \mathrm{I}-\mathrm{O}^{2+}\left(2 p^{2}{ }^{3} P\right) \rightarrow \mathrm{O}^{+}\left(2 s^{2} 2 p^{3}{ }^{4} S\right)$, $\mathrm{II}-\mathrm{O}^{2+}\left(2 p^{2}{ }^{3} P\right) \rightarrow \mathrm{O}^{+}\left(2 s^{2} 2 p^{3}{ }^{2} D\right)$, III $-\mathrm{O}^{2+}\left(2 p^{2}{ }^{3} P\right) \rightarrow$ $\mathrm{O}^{+}\left(2 s^{2} 2 p^{3}{ }^{2} P\right), \mathrm{IV}-\mathrm{O}^{2+}\left(2 p^{2}{ }^{3} P\right) \rightarrow \mathrm{O}^{+}\left(2 s 2 p^{4}{ }^{2} P\right), \mathrm{V}-$ $\mathrm{O}^{2+}\left(2 p^{2}{ }^{1} D\right) \rightarrow \mathrm{O}^{+}\left(2 s^{2} 2 p^{3}{ }^{2} D\right), \quad \mathrm{VI}-\mathrm{O}^{2+}\left(2 p^{2}{ }^{1} S\right) \rightarrow$ $\mathrm{O}^{+}\left(2 s^{2} 2 p^{3}{ }^{2} D\right), \mathrm{VII}-\mathrm{O}^{2+}\left(2 p^{2}{ }^{1} S\right) \rightarrow \mathrm{O}^{+}\left(2 s 2 p^{4}{ }^{4} P\right)$. 
$\mathrm{CO}^{+}\left(X^{2} \Sigma^{+}, v=0\right)$ в низшем колебательном состоянии, что и объясняет большое сечение захвата одного электрона с образованием недиссоциированного молекулярного иона (процесс (1)).

При анализе величин дефектов энергии процессов с участием двухзарядных ионов $\mathrm{C}^{2+}, \mathrm{N}^{2+}$ и $\mathrm{O}^{2+}$ надо учитывать, во-первых, что в ионном пучке не полностью ободранные ионы могут находиться как в основном, так и в метастабильных состояниях [26-29]. Во-вторых, процесс одноэлектронного захвата может сопровождаться изменением электронного состояния остова иона, захватившего электрон, т.е. осуществляться как многоэлектронный процесс, даже если не происходит возбуждения образующегося молекулярного иона.

На рис. 6, $a$ приведены дефекты энергии для наиболее вероятных каналов процесса захвата одного электрона ионами $\mathrm{C}^{2+}$. Как видно из приведенных данных, при взаимодействии иона $\mathrm{C}^{2+}\left(2 s^{2}{ }^{1} S\right.$ ) (канал I на рис. $6, a$ ) с молекулой $\mathrm{CO}$ в „окно реакции“ попадают лишь каналы, ведущие к образованию ионов в состоянии $\mathrm{C}^{+}\left(2 s^{2} 2 p^{2} P\right)$ и недиссоциированных ионов $\mathrm{CO}^{+}\left(A^{2} \Pi\right)$ и $\mathrm{CO}^{+}\left(\mathrm{B}^{2} \Sigma^{+}\right)$. Остальные пять рассматриваемых каналов процесса захвата электрона (отмечены на рис. 6, $a$ римскими цифрами II-VI), осуществляются при взаимодействии метастабильного иона $\mathrm{C}^{2+}\left(2 s 2 p{ }^{3} P\right)$ с молекулой. Канал процесса захвата II с образованием иона $\mathrm{C}^{+}\left(2 s^{2} 2 p^{2} P\right)$ в основном состоянии, должен, как видно из рис. $6, a$, сопровождаться диссоциацией образующегося молекулярного иона $\mathrm{CO}^{+}$. Результаты проведенного качественного анализа подтверждаются исследованием заселенности электронных состояний партнеров процесса захвата одного электрона при столкновении ионов $\mathrm{C}^{2+}$ с энергией $E_{p} \approx 1 \mathrm{keV}$ с молекулами $\mathrm{CO}$, выполненным методом столкновительной спектроскопии [26]. Дефекты энергии каналов процесса захвата, выделенные жирными линиями на рис. 6, $a$, наблюдаются в настоящей работе, что подтверждает обоснованность используемого метода качественного анализа.

При анализе взаимодействия ионов $\mathrm{O}^{2+}$ с молекулами СО учитывалось, что ионы-снаряды могли находиться в трех электронных состояниях $\mathrm{O}^{2+}\left(2 s^{2} 2 p^{2} 3 P\right)(\mathrm{GS})$, $\mathrm{O}^{2+}\left(2 s^{2} 2 p^{2} 1 D\right)(\mathrm{MS} \Delta E=2.51 \mathrm{eV})$ и $\mathrm{O}^{2+}\left(2 s^{2} 2 p^{2}{ }^{1} S\right)$ (MS $\Delta E=5.35 \mathrm{eV}$ ) [24]. Как видно из приведенных на рис. $6, b$ дефектах энергии каналов процесса захвата, при захвате одного электрона ионами $\mathrm{O}^{2+}$ в „окно реакции“ попадают каналы процессов, в основном приводящие к диссоциации образующегося молекулярного иона $\mathrm{CO}^{+}$. Молекулярные ионы $\mathrm{CO}^{+}\left(\mathrm{C}^{2} \Delta\right)$ и $\mathrm{CO}^{+}$(D $\left.{ }^{2} \Pi\right)$, которые в рамках рассматриваемой модели, вероятно, образуются при реализации канала процесса захвата III, имеют небольшую глубину потенциальной ямы [22] и, скорее всего, диссоциируют. Каналы процесса захвата, обозначенные на рис. $6, b$ IV и VII должны приводить к образованию стабильных молекулярных ионов, но эти процессы обусловлены возбуждением остова молекулярного иона $\mathrm{O}^{+}$, что должно приводить к уменьшению сечения этих процессов по сравнению с одноэлектронными.

\section{Заключение}

Таким образом, структура электронных уровней иона $\mathrm{O}^{+}$, образующегося в процессе захвата, и определяет доминирующую роль процесса диссоциативного захвата одного электрона. Качественный анализ, основанный на рассмотрении дефекта энергии процесса, показывает, что для однозарядных налетающих ионов этот эффект должен наблюдаться для ионов $\mathrm{He}^{+}$[30] и для $\mathrm{Ne}^{+}$, для которых это и показано экспериментально [8]. Кроме того, для указанных выше ионов следует ожидать доминирующей роли процесса диссоциативного захвата при взаимодействии с молекулами $\mathrm{N}_{2}$ и $\mathrm{O}_{2}$. Ясно, что для многозарядных ионов $A^{z+}$ с $z \geq 5$ из-за большого количества конечных электронных состояний, образующегося в процессе захвата иона $A^{(z-1)+}$, процесс (1) будет доминировать над процессом (2), что и наблюдалось нами при исследовании взаимодействия ионов $\mathrm{Ar}^{z+}(z=5,6)$ с молекулами СО.

\section{Финансирование работы}

Работа выполнена по Гос. заданию тема № 0040-2019-0023.

\section{Конфликт интересов}

Авторы заявляют, что у них нет конфликта интересов.

\section{Список литературы}

[1] Rudd M.E., Goffe T.V., Itoh A., Du Bois R.D. // Phys. Rev. A. 1985. Vol. 32. P. 829-835.

[2] Rudd M.E., Goffe T.V., Itoh A. // Phys. Rev. A. 1985. Vol. 32. P. 2128-2133.

[3] АЯросимов В.В., Лейко Г.А., Мамаев Ю.А., Панов М.Н., Вуйович М. // ЖЭТФ. 1973. Т. 65. Вып. 2. (8). С. 495-504.

[4] Shah M.B., Gilbody H.B. // J. Phys. B: At. Mol. Opt. Phys. 1990. Vol. 23. P. 1491-1499.

[5] Latimer C.J. // J. Phys. B: Atom. Molec. Phys. 1977. Vol. 10. P. 515-522.

[6] Orient O.J., Srivastava S.K. // J. Phys. B: At. Mol. Phys. 1987. Vol. 20. P. 3923-3936.

[7] Tian C., Vidal C.R. // Phys. Rev. A. 1999. Vol. 59. P. 1955-1965.

[8] Graham W.G., Latimer C.J., Browning R., Gilbody H.B. // J. Phys. B: At. Mol. Phys. 1973. Vol. 6. P. 2641-2652.

[9] Lindsay B.G., Yu W.S., Stebbings R.F. // Phys. Rev. A. 2005. Vol. 71. P. 032705

[10] Абросимов В.В., Басалаев А.А., Огуриов Г.Н., Панов М.Н. // ЖТФ. 2014. Т. 84. Вып. 5. С. 14-20.

[11] Афросимов В.В., Басалаев А.А., Панов М.Н. // Письма в ЖТФ. 2017. Т. 43. Вып. 2. С. 76-83.

[12] Гурвич Л.В., Карачевцев Г.В., Кондратьев В.Н., Лебедев Ю.А., Медведев В.А., Потапов В.К., Ходеев Ю.С. Энергия разрыва химических связей. Потенциалы ионизации и сродство к электрону. М.: Наука, 1974. 351 с.

[13] Афросимов В.В., Басалаев А.А., Морозов Ю.Г., Панов М.Н., Смирнов О.В., Тропn Э.А. // ЖТФ. 2011. Т. 81. Вып. 5. С. 9-15. 
[14] Čadež I., Greenwood J.B., Chutjian A., Mawhorter R.J., Smith S.J., Niimura M. // J. Phys. B: At. Mol. Opt. Phys. 2002. Vol. 35. P. 2515-2524.

[15] Kusakabe T., Miyamoto Y., Tawara H. // Phys. Rev. A. 2006. Vol. 73. P. 022706.

[16] Graham W.G., Latimer C.J., Browning R., Gilbody H.B. // J. Phys. B: At. Mol. Phys. 1974. Vol. 7. P. L405-L408.

[17] Bene E., Martíanez P., Halsáz G.J., Vibók Á., BacchusMontabonel M.C. // Phys. Rev. A. 2009. Vol 80. P. 012711.

[18] Gabás P.M.M., Errea L.F., Méndez L., Rabadán I. // Phys. Rev. A. 2012. Vol. 85. P. 012702.

[19] Salop A., Olson R.E. // Phys. Rev. A. 1976. Vol. 13. P. $1312-1320$.

[20] Krupenie P.H., Weissman S. // J. Chem. Phys. 1965. Vol. 43. P. $1529-1534$.

[21] Krishnamurthi V., Nagesha K., Marathe V.R., Mathur A. // Phys. Rev. A. 1991. Vol. 44. P. 5460-5467.

[22] Okada K., Iwata S. // J. Chem. Phys. 2000. Vol. 112. P. $1804-1808$.

[23] Hierl P.M., Franklin J.L. // J. Chem. Phys. 1967. Vol. 47. P. 3154-3161.

[24] Kramida A., Ralchenko Yu., Reader J. NIST ASD Team (2015). NIST Atomic Spectra Database (ver. 5.3), [Online]. Available: http://physics.nist.gov/asd

[25] Janev R.K., Presnyakov L.P. // Phys. Rep. 1981. Vol. 70. N 1.

[26] Leputsch P., Dumitriu D., Aumayr F., Winter H.P. // J. Phys. B: At. Mol. Opt. Phys. 1997. Vol. 30. P. 5009-5024.

[27] Jellen-Wutte U., Schweinzer J., Vanek W., Winter H. // J. Phys. B: At. Mol. Phys. 1985. Vol. 18. P. L779-L785.

[28] Kamber E.Y., Brenton A.G., Beynon J.H. // J. Phys. B: At. Mol. Phys. 1984. Vol. 17. P. 4919-4933.

[29] Абросимов В.В., Басалаев А.А., Панов М.Н. // ЖТФ. 2005. T. 75. Вып.8. С. 26-31. [Afrosimov V.V., Basalaev A.A., Panov M.N. // Tech. Phys. 2005. Vol. 50. N 8. P. 987.]

[30] Werbowy S., Pranszke B. // Phys. Rev. A. 2016. Vol. 93. P. 022713. 